\title{
Butting heads
}

\author{
A recent controversy on sport-related dementia underscores the need for comprehensive epidemiology studies.
}

A ccumulating evidence has suggested that professional players of American football might be at increased risk of early-onset dementia. This October, a string of heated debates on this issue culminated in a US congressional House Judiciary Committee hearing in which the National Football League (NFL) denied a possible link between concussions, chronic traumatic encephalopathy (CTE) and other dementia-related neurodegenerative disorders, despite scientific evidence to the contrary. As an antitrust-exempted franchise that generates billions of dollars annually, the NFL has a deep financial stake in this issue. However, although few would disagree with the notion that repeated head trauma is likely to have bad consequences, there is surprisingly little epidemiological data on the prevalence, both among the general public and in professional athletes, or the factors that could potentially increase the risk of CTE. Instead of denying culpability, the league should stop stonewalling and should aid comprehensive studies that help define the exact neurological risks of playing the sport and work with medical professionals to refine the sport so as to make it safer for professionals and amateurs alike.

CTE is a relatively rare neurodegenerative disease with progressive dementia and extensive tau-immunoreactive neurofibrillary tangles throughout the brain. Originally identified in boxers, it is linked to multiple bouts of head injury and its clinical symptoms can be very similar to those of other dementia-linked disorders, such as Alzheimer's disease. As with many other neurodegenerative diseases, definitive diagnosis can only be made on postmortem inspection. A limited number of single case studies from postmortem examination of former football players and retrospective cohort studies of boxers have bolstered the plausible association between the two phenomena, but there have been no long-term epidemiology studies to address this issue.

By design, epidemiology studies cannot establish causality; they can only identify risk factors for a given disease. Nevertheless, the correlations seen in such studies could provide a wealth of information and help seed future experiments that could explain the disease etiology. Designing and executing a good epidemiology study can be extremely difficult, particularly when it comes to neurodegenerative disorders. For example, the University of Michigan conducted a study (which has yet to be peer-reviewed and published) that reports a higher incidence of dementia and cognitive deficits in former NFL players. However, because this study was based on telephone interviews that retrospectively surveyed subject-reported health history, one caveat is that this design may cause selective self-reporting and increase potential bias on the basis of the specific questions being asked. In addition, the accuracy of subjects remembering correct information or reporting pertinent details could be questionable if they suffer from memory decline in the first place.

The best epidemiology studies use a prospective design rather than a retrospective one. Ideally, a group of subjects are monitored by healthcare professionals over many years, allowing accurate diagnoses of disease progression and factoring in any diagnostic variability. An example of one such study is the recently completed Alzheimer's Disease Neuroimaging Initiative (ADNI), which provided a 5-year longitudinal study of 800 elderly volunteers at various stages of cognitive decline. Even ADNI, however, comes with caveats, as the timing and duration of the study is critical. How early and how long should the subjects be monitored? For many disorders such as Parkinson's or Alzheimer's, there are neuronal changes that can occur long before the subjects show symptoms, and catching subjects early enough may also be difficult. Diagnostic parameters that may cause subtle brain changes may be missed entirely if the patients are not studied early enough.

Despite the potential design issues and interpretational caveats, good epidemiology studies can inspire basic scientists and open new research avenues. An epidemiology study, for example, linked pesticide exposure to an increased rate of idiopathic Parkinson's disease ${ }^{1}$, which was instrumental in creating a rodent model of Parkinson's disease ${ }^{2}$ and investigating mitochondrial dysfunction in this disorder. Ideally, however, epidemiologists and basic scientists should work in concert to exchange information and help spur each other's research efforts. As Richard Mayeux, an epidemiologist working on Alzheimer's research at Columbia University notes, meetings that bring together molecular, genetic and epidemiology researchers have been useful to refine study parameters and to learn about risk factors that can be applied to improve molecular or genetic models of disease.

As for the current debate of whether football-related head injuries are a risk factor for CTE, the statistics-obsessed NFL has a wealth of information that could be tapped to help address this gap. The NFL keeps extensive records on each player's performance and injuries (neurological or otherwise) and has the necessary resources to fund regular and independent evaluations of the health of its players. At the recent congressional hearing, the NFL promised to make such data available. It will be essential to have this material evaluated by scientists who are not affiliated with either the league or the players' advocacy group to avoid the NFL's and professional players' intrinsic conflicts of interest. A prospective large-scale epidemiological study may not be possible using just professional football players, but a case-cohort comparison comparing football players with other professional athletes who do not undergo as many head collisions as football players do (such as baseball players) would be useful. Instead of trying to discredit the existing evidence, the NFL and other professional sports organizations would do well to help scientists, clinicians and the public better understand and prevent sports-associated risks in the first place.

1. Butterfield, P.G., Valanis, B.G., Spencer, P.S., Lindeman, C.A. \& Nutt, J.G. Neurology 43, 1150-1158 (1993).

2. Betarbet, R. et al. Nat. Neurosci. 3, 1301-1306 (2000). 